\title{
Spinal cord pencil-shaped softening: comparison between the clinical findings and the autopsy findings. Case report
}

\author{
T Muramatsu MD, S Kikuchi MD, E Watanabe MD \\ Department of Orthopaedic Surgery, Fukushima Medical College, Fukushima City, \\ 960-12, Japan.
}

\begin{abstract}
The neurological symptoms and the magnetic resonance imaging (MRI) findings in a patient with a spinal pencil-shaped form of softening were compared with the autopsy findings. The distribution and shape of the lesion on MRI, especially on T2-weighted images, correlated well with the autopsy findings at the level between the medulla oblongata and the T2 segment. But these findings did not necessarily correlate with the neurological abnormalities. A pencilshaped lesion that had extended cephaladly, and which was seen on MRI and at autopsy, did not manifest neurological deficit. MRI is useful to aid the clinical diagnosis of spinal cord pencil-shaped softening.
\end{abstract}

Keywords: spinal cord pencil-shaped softening; MRI; autopsy; clinical neurological syndrome.

\section{Introduction}

Spinal cord pencil-shaped softening is a peculiar cavitated lesion that occurs in the ventral part of the dorsal funiculus and extends longitudinally over several segments of the spinal cord. ${ }^{1-3}$ This condition is associated with posttraumatic myelopathy, malignant tumours, adhesive arachnoiditis and many other spinal diseases. Although it has been diagnosed by autopsy, clinical diagnostic criteria have not yet been established. Recently, magnetic resonance imaging (MRI) has demonstrated small lesions of the spinal cord. ${ }^{4-7}$ In this report, we describe the neurological symptoms and MRI findings in a patient with spinal cord pencil-shaped softening in comparison with the autopsy findings.

\section{Case report}

A 65 year old man was admitted with a chief complaint of pain in the nape of the neck and tetraplegia after having been intoxicated the night before. He was anaesthetic below the C6 spinal segment and exhibited hypalgesia at the C5 segment. The manual muscle testing (MMT) revealed a score of grade 1 for the deltoid muscles and 0 for all the muscles distal to the biceps. The deep tendon reflexes were normal in the extremities and the abdomen. There were no abnormal reflexes. The patient's respirations were normal, and he was fully conscious. The cerebro-spinal fluid was clear. No abnormalities were found in the plain radiographs or in a CT scan of the cervical spine and head. Five days after admission, muscle power in the trapezius and sternocleidomastoid muscles diminished. Diaphragmatic movement and the level of consciousness decreased as well. Mechanical ventilation was instituted. Consciousness recovered completely and muscle power recovered somewhat over the next 2 weeks. The neurological symptoms stabilised 1 month after admission (Fig 1). The upper and

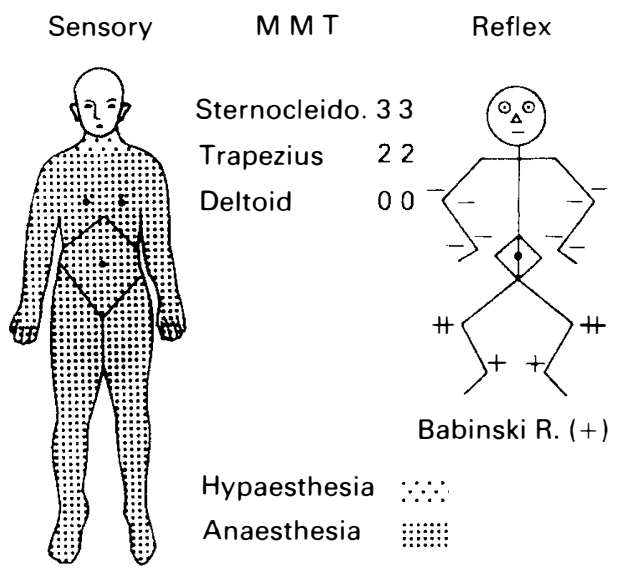

Figure 1 Neurological findings (static stage). 
lower extremities and the body below the clavicles were anaesthetic: hypalgesia of the lower neck was present. Muscle power was completely lost in all of the muscles below the deltoid muscles (MMT 0), and the sternocleidomastoid muscles showed an MMT of 3. The deep tendon reflexes were lost in the upper limbs, and increased in the lower limbs. These clinical findings suggested a spinal cord lesion at the $\mathrm{C} 4$ spinal segment. The paralysis lasted for 1 year, at which time the patient died from pneumonia.

\section{MRI findings (Fig 2)}

T1-weighted images showed a mild intensity signal of the spinal cord between the C3-4 and C6-7 disc levels. T2-weighted images revealed a high intensity signal of the spinal cord between the levels of the C3 and C6 vertebrae, including a low intensity signal in the cord at the $\mathrm{C} 4-5$ disc level. The high intensity image extended as a long, narrow lesion extending to the medulla oblongata and to the level of the $\mathrm{T} 1$ vertebra. The cephalad lesion was in the right side of the spinal cord and the caudal lesion was in the left side. These were interpreted as representing extensive necrosis and oedema of the spinal cord between the levels of the $\mathrm{C} 3$ and $\mathrm{C} 6$ vertebrae, accompanied by spotty haemorrhage at the C4-5 disc level, with longitudinally expanding necrosis and oedema that suggested pencil-shaped softening.

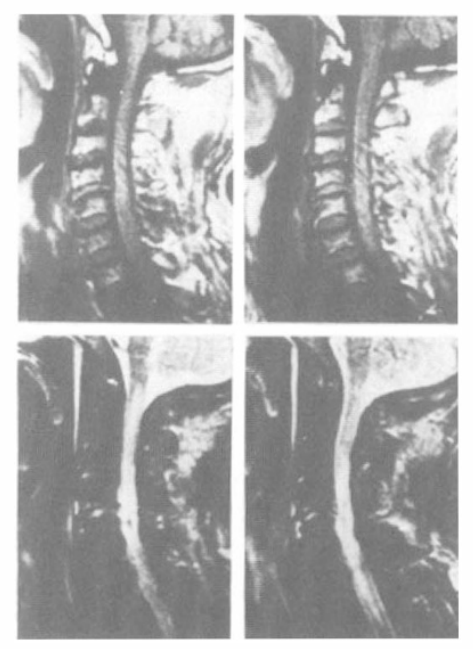

Figure 2 MRI: the upper images are T1weighted, the lower images are T2-weighted. the left images are right side of the spinal cord. and the right images are left side of the spinal cord.

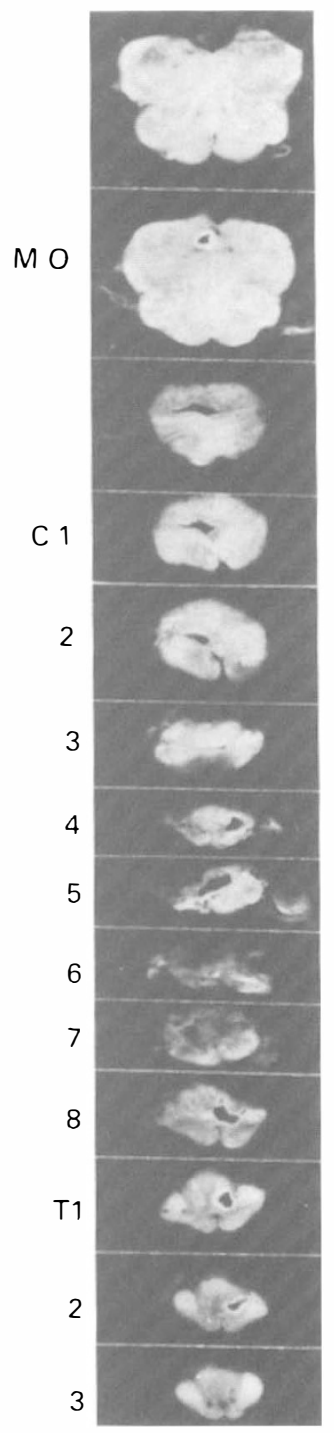

Figure 3 Segmental cross section of the spinal cord. The $\mathrm{C} 4-\mathrm{C} 7$ segments are atrophied, the medulla oblongata-C2 segments and the C7-T2 segments show pencil-shaped cavities.

\section{Autopsy findings (Figs 3 and 4)}

The spinal cord showed atrophy between the C4 and C7 segments (between the C3 and C6 vertebrae), and necrosis was found in the $\mathrm{C} 6$ segment (at the C4-5 disc level). A macroscopic observation of cross sections of spinal cord segments revealed extensive softening and a secondary syringomyelia in the areas of the lesions. Softening and cavities extended in a pencil shape to the medulla oblongata in the 


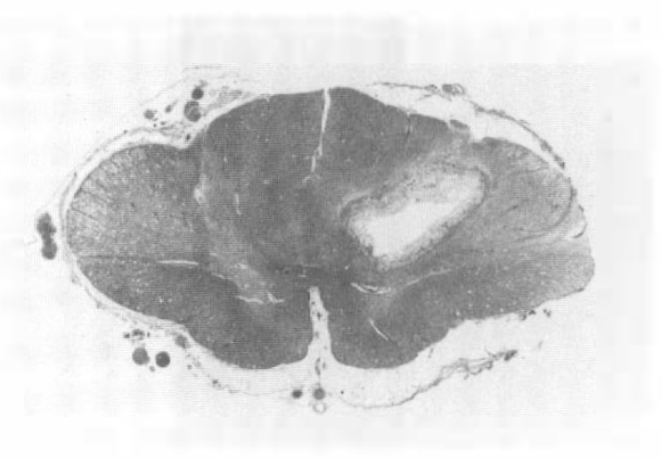

Figure 4 Histology of pencil-shaped softening of T2 segment. Softening cavity is present in the ventral portion of the left dorsal funiculus.

right side of the spinal cord and to the $\mathrm{T} 2$ segment (at the $\mathrm{T} 1$ vertebra) on the left side. Histologically, normal tissues of the C6 segment had been replaced completely by granulation tissue accompanied by cavities. The granulation tissue showed extensive infiltration of macrophages and deposits of haemosiderin. Pencilshaped softening was present on the ventral side of the dorsal funiculus. A large number of lipid-rich macrophages had infiltrated the wall, but there was little deposition of haemosiderin. In spite of softening, neither the spinal arteries nor the veins were obliterated.

\section{Discussion}

The correlation between neurological symptoms, MRI findings and autopsy findings, is shown in Figure 5. The MRI study and the autopsy showed softening and cavitation between the $\mathrm{C} 4$ and $\mathrm{C} 7$ segments, and neurological abnormalities were found in these segments and caudally. A pencilshaped softening that extended cephaladly was found on the MRI and at autopsy, but did not result in neurological abnormalities. Kikuchi et al have reported that an upward extension of transient, reversible paralysis can occur following the sudden onset of paralysis and coincides with areas of pencilshaped softening found at autopsy. ${ }^{1}$ In our case, the paralysis extended upward 5 days

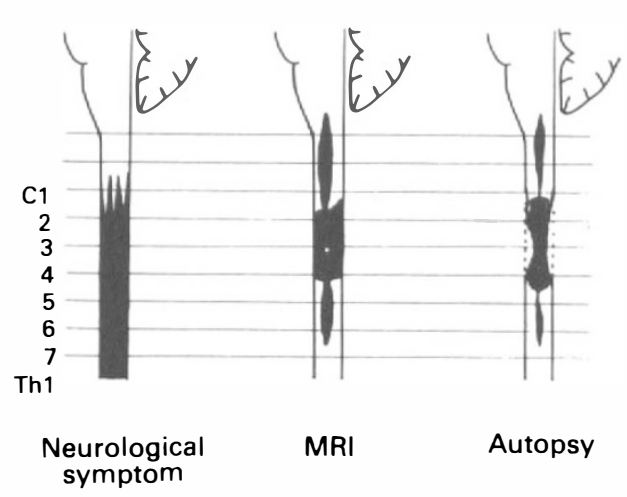

Figure 5 Comparison of neurological manifestations, MRI and autopsy findings.

after admission and was partially irreversible. The level of irreversible paralysis coincided with the area of extensive softening. An upward extension of the paralysis was seen in only a part of the area of pencil-shaped softening found at autopsy. These findings suggest that pencil-shaped softening does not necessarily produce a neurological abnormality, and we consider that it depends upon the site and size of the softening. The abnormalities found on MRI coincided with the autopsy findings morphologically. An autopsy was performed 1 year after the MRI, but the fact that the autopsy findings did correlate with the MRI findings clearly shows that MRI, especially the T2weighted images, is useful for the clinical diagnosis of pencil-shaped softening.

\section{Conclusion}

The distribution and shape of the spinal cord lesion seen on MRI, especially on T2-weighted images, coincided with autopsy findings in spinal pencil-shaped softening, but not necessarily with the neurological abnormalities. MRI appears to be useful in diagnosing pencil-shaped softening of the spinal cord, although the anatomical distribution does not necessarily correlate perfectly with the clinical presentation.

\section{References}

1 Kikuchi S, Hasue M, Takemura T, Inoue K (1984) Clinical significance of spinal pencil-shaped softening of the spinal cord. Seikei-Geka 35: 289-294 (in Japanese). 
2 Kume A, Takahashi A, Hashizume Y (1989) Spinal pencil-shaped softening. Report of an autopsy case study using a three-dimensional model. Acta Pathol Jpn 39: 135-140.

3 Nagashima K, Shimamine T (1974) Anatomo-pathologic study of 'pencil-shaped softening' of spinal cord. Shinkei-Shinpo 18: 153-166 (in Japanese).

4 Bodurant FJ, Cotlor HB, Kulkarni MV, McArdle CB, Harris JH Jr (1990) Acute spinal cord injury. A study using physical examination and magnetic resonance imaging. Spine 15: 161-168.

5 Fisher R, Zanella F, Lanfermann H (1993) The dynamics of magnetic resonance findings in patients with a spinal haematoma. Paraplegia 31: 180-185.

6 Sett P, Crockard HA (1991) The value of magnetic resonance imaging (MRI) in the follow-up management of spinal injury. Paraplegia 29: 396-410.

7 Weirich SD, Cotlor HB, Narayana PA, Hazle JD, Jackson EF, Coupe KJ et al (1990) Histopathologic correlation of magnetic resonance imaging signal patterns in a spinal cord injury model. Spine 15: 630-638. 